\title{
Loss of position after initial manipulation of distal radius fractures in elderly
}

\begin{abstract}
A retrospective analysis was carried out on the loss of position after initial manipulation of the distal radius fractures at Dallah Hospital, Riyadh. Forty patients, above 60 years old, were selected from 2013-2015. Three measurements were taken on the X-rays of the injured wrist at three different occasions. Radial length and radial inclination were measured on the $\mathrm{AP}$ and volar tilt was measured on the lateral X-ray. X-rays were measured at the time of injury, soon after manipulation and at first follow-up at about a week. Assessment was done only from the patients X-rays and notes.
\end{abstract}

The criteria used to label loss of position were loss of 10 degrees in volar tilt, loss of 5 degrees in radial inclination and loss of $2 \mathrm{~mm}$ in radial length. The total number of patients who lost position after manipulation was $23(57.5 \%)$. All the three measurements were lost in $4(10 \%)$ patients. Volar tilt was lost in $17(42.5 \%)$ cases, radial length was lost in 15 $(37.5 \%)$ cases and radial inclination was lost in $7(17.5 \%)$ cases. Statistical tests showed $p$ $<0.0001$ for volar tilt and radial length and $\mathrm{p}<0.05$ for radial inclination.

Patients who were given a complete cast within twenty four hours lost position in $7(23.3 \%)$ cases. The ten patients whose cast was completed later than twenty four hours but within a week had all lost their positions.

We concluded that the elderly patients should be manipulated and not left alone as most of the time we can achieve good reduction. The reason for lost reduction is more likely to be the dorsal slab and that they should be given a complete cast / Charnley method of radial slab.

Keywords: Distal radius fracture, Instability, Osteoporosis, Elderly, Radial Tilt, Eaematoma
Volume 7 Issue 2 - 2017

\author{
Nadeem Khalid \\ Dallah Hospital, Saudi Arabia
}

Correspondence: Nadeem Khalid,Villa A-28, Dallah Hospital, PO Box 87833, Riyadh II653, Saudi Arabia

Email amirhosseinkesavarz@aol.com

Received: December 04, 2016 | Published: January 19, 2017

\section{Introduction}

Distal radial fractures account for almost $17 \%$ of all fractures encountered in Accident and Emergency Department. ${ }^{1}$ There is a bimodal age distribution for this fracture, with one peak in the first decade and the second peak in the sixth decade. More frequently these fractures occur in women than in men and usually occur with low impact falls in the elderly group.

In 1814 Abraham Colles'. ${ }^{2}$ first described this fracture and since then there has been a continuous controversy about the best method of treatment. The standard of treatment for most fractures of the distal radius has been closed reduction and immobilization. Additionally, the predominance of injury to the elderly population, often with coexisting medical problems, low functional demands and significant osteoporosis has helped to further establish closed management as the treatment of choice. Colles' ${ }^{3}$ opined that deformity does not correlate with functional limitation and this is still widely believed among orthopaedic surgeons.

Although Colles' fracture can usually be reduced very easily, it is difficult to maintain the reduction in a plaster cast, especially when it is commented. Gartland and Werley in $1951 .^{3}$ reported $60 \%$ of their patients healed in an unreduced position.

There is not an agreement in literature about adequate reduction, but it has been suggested that a radial tilt of greater than 10 degrees is important. A volar tilt of at least 0 degrees, that is, no dorsal tilt and shortening of no more than $2 \mathrm{~mm}$ is evidence of satisfactory reduction.

Fractures can re-displace during immobilization in a cast or splint and re-manipulation is a common practice, yet little has been written about the incidence and causes of displacement after initial manipulation.

\section{Methods and materials}

We undertook a retrospective study of the notes and radiographs of the patients who attended the Accident and Emergency Department of Dallah Hospital, Riyadh, with a distal radius fracture. We identified sixty patients in the period from 2013-2015 who needed to have manipulation of their distal radius fracture under haematoma block and observed forty patients whose notes and X-rays were available to us. Details of the patients were collected from the Emergency Room (ER) and Plaster Room register. Then medical notes and X-rays were traced from the Medical Records Department.

The following data was collected. The age, the sex, the side of the injury, the cause of the injury and the type of back slab applied (below or above elbow). The radiographs were all reviewed by a single examiner. Fractures were classified by AO classification system. Radiographic assessment was done by measuring the radial inclination, radial length and the volar tilt. Radial inclination and radial length was measured on the AP X-ray and volar tilt measured on the lateral X-ray. All the X-rays were taken by a standard method. Measurements were taken on 3 occasions. First at the time of injury, second just after the manipulation and finally when the patients came for follow-up for the first time in the fracture clinic. The criteria used to label loss of position were loss of 10 degrees or more in volar tilt, loss of 5 degrees or more in radial inclination and loss of $2 \mathrm{~mm}$ or more in radial length. 
All the patients were over sixty years of age and were sent home the same day, after the manipulation in ER. Manipulation was done under haematoma block. They were given a below elbow plaster back slab and a collar and cuff sling to protect the fracture. Thirty patients were seen within twenty four hours for completion of the cast and then followed up in regular fracture clinic within a week. The other ten patients were followed up within 3-15 days for unknown reasons.

At the first follow-up appointment in fracture clinic, final measurement was taken and results were evaluated as the percentage of patients whose position has been changed since the initial manipulation, which of the 3 measurements has been changed and whether the change was significant enough to perform another procedure or not. All the measurements were repeated twice at an interval of one week to minimise intra-observer error.

\section{Results}

This is a retrospective study of forty patients who attended ER of Dallah Hospital, Riyadh, from year 2013-2015 for distal radius fractures. All the patients had manipulation under haematoma block and were given a below elbow back slab for protection of the fracture. The age of the patients varied between 61 years to 90 years, mean age 74 years. All the patients were injured by low energy trauma by having a fall on the ground due to ice, tripped on something or fell from the chair

There were 39 females and 1 male only. Left side was involved in 25 patients and Right side was involved in 15.

The most common fracture type found was A2 followed by A3. Only one fracture was type $\mathrm{B} 3$ and few were $\mathrm{C} 1$ and $\mathrm{C} 2$. No fracture was of type A1, B1, B2 and C3 type (Table 1).

Table I Number of patients according to AO Classification

\begin{tabular}{ll}
\hline Classification by AO System & Number of Patients \\
\hline Al & 0 \\
A2 & 16 \\
A3 & 14 \\
BI & 0 \\
B2 & 0 \\
B3 & 1 \\
Cl & 6 \\
C2 & 3 \\
C3 & 0 \\
\hline
\end{tabular}

Observations for volar tilt, radial length and radial inclination

Forty patients were observed and angles for volar tilt, radial length and radial inclination were measured at the time of injury, soon after manipulation and at about one week time at first follow-up. Thirty patients were seen within twenty four hours, on the next day for completion of the cast and ten patients were seen within 3-15 days, mean 8 days for follow-up and completion of cast.

The criteria used to label loss of position were loss of 10 degrees in volar tilt, loss of 5 degrees in radial inclination and loss of $2 \mathrm{~mm}$ in radial length. All the measurements were taken twice at an interval of 1 week and done by the same examiner to delete intra-observer error. Statistical examination proved no significant intra-observer error between the two readings.

The total number of patients who lost position after manipulation was $23(57.5 \%)$. All the three measurements were lost in $4(10 \%)$ patients. Volar tilt was lost in $17(42.5 \%)$ cases, radial length was lost in $15(37.5 \%)$ cases and radial inclination was lost in $7(17.5 \%)$ cases.
Because most of the measurements of volar tilt, radial length and radial inclination at the three intervals were not normally distributed, results were reviewed statistically by both parametric and non-parametric tests. Friedman test for differences between the three measurements of pre and post manipulation and also after a week and Wilcox son test for pair wise investigations to detect the overall significance. Both the tests showed $\mathrm{p}<0.0001$ for volar tilt and radial length and $\mathrm{p}<0.05$ for radial inclination.

Patients who were given a complete cast within twenty four hours lost position in 7 (23.3\%) cases. Two patients had re-manipulation with K-wiring, two patients accepted their deformity and function, in two patients the deformity and function were accepted by the surgeon due to medical complications as they were not fit for surgery and one patient had a re-fracture in three weeks time and therefore ended with a very stiff wrist which was accepted by the surgeon and the patient.

The ten patients whose cast were completed later than twenty four hours but within a week had all lost their initial position. Three patients were re-manipulated and given an external fixator, five patients were offered a distal radial osteotomy but all of them rejected the offer and accepted the deformity. Two patients were not offered any further surgery because of their medical condition (Table 2).

Table 2 Consolidation of results

\begin{tabular}{ll}
\hline Total Number of Patients & $\mathbf{4 0}$ \\
\hline Patient's who Lost their Position & $23(57.5 \%)$ \\
All Three Measurements Lost & $04(10 \%)$ \\
Volar Tilt Lost & $17(42.5 \%)$ \\
Radial Length Lost & $15(37.5 \%)$ \\
Radial Inclination Lost & $07(17.5 \%)$ \\
Position Lost in Patients with Complete Cast within 24 Hours & $07(23.3 \%)$ \\
Cast Completed Later than 24 Hours & 10 \\
Position Lost in Patients with Complete Cast Later than 24 Hours & $10(100 \%)$
\end{tabular}

\section{Discussion}

A distal radius fracture involves the metaphysic of the radius and potentially the radiocarpal and radioulnar joints. The deformities produced by distal radius fracture are dependent on the degree of combinations and articular involvement of the radiocarpal and radioulnar joints and also on the stability of the distal radioulnar joint. The severity of the injury affects the anatomic and functional outcome.

There are four principles in the treatment of distal radius fractures. ${ }^{4}$

A. Restoration of articular surface and axial alignment

B. Maintenance of reduction

C. Achievement of union and

\section{Restoration of hand and wrist function.}

These principles may be varied to an extent depending on patient variables such as low functional demand, significant medical illness, or inability to comply with post-operative instructions, all of which may justify acceptance of the deformity. This was also shown in our study that four patients despite having a re-collapse of the fracture after initial manipulation were left alone, as the medical condition of the patients did not allow any further surgery.

Authors disagree on what an adequate reduction means but review of literature suggests

The following criteria for an acceptable reduction. ${ }^{4}$

A. Change in volar tilt of no more than 10 degrees

B. Radial shortening of no more than $2 \mathrm{~mm}$ and 


\section{Change in radial inclination of no more than 5 degrees.}

D. When fracture is intra-articular, no more than $2 \mathrm{~mm}$ of articular step-off should be accepted.

Reduction may be attempted under local/regional anaesthesia (haematoma / Bier's block). It has been shown by Abbaszadegan \& Jonsson. ${ }^{5}$ that these fractures are best reduced under regional (Bier's block) or general anaesthesia when compared to a haematoma block. Unfortunately facility of Bier's block was not available at Dallah Hospital therefore haematoma block was used in all the forty cases involved in our study. Reduction should be achieved primarily by longitudinal traction with reduction of the specific deformity present through manual pressure over the fracture fragments. If reduction is not achieved then formal surgical management is indicated.

When we studied the results of reduction, we found no correlation between fracture classification, initial displacement and the final radiographic outcome. More than $50 \%$ of the cases lost reduction in the first week of immobilization. This is very similar to the findings of reduction in older patients by Beamer and McQueen et al. ${ }^{6}$ who showed that even if a satisfactory reduction is achieved in most cases, it is not possible to maintain this position in the elderly patient and 37 out of $4(84 \%)$ patients lost reduction in their study.

Van der Linden and Ericson et al. ${ }^{7}$ describes somewhat different results as they found that the volar tilt (dorsal angle) is highly dependent on the original injury and also that if the volar tilt is restored, shortening tends to diminish.

We also noticed that loss of reduction in patients who had their cast completed within twenty four hours, usually lead to an "acceptable" position given the age and demands of the patients, but all the ten patients who had the cast completed later than twenty four hours but within a week, all had a significant loss of reduction and were given re-reduction under general anaesthesia. Again this finding is not supported by Van der Linden and Ericson et al. ${ }^{7}$ who compared five different types of the below-elbow plaster cast immobilization and found that the anatomical and functional results were similar, whatever the type of immobilization used.

Looking at our results of the ten patients who were not given a completed cast in the first twenty four hours and therefore lost reduction in all of them, we tend to agree with Sir John Charnley who describes a reliable method of putting a plaster cast after reduction of Colles' fracture in his book "The Closed Treatment of Common Fractures". He stated that for mechanical reasons the ideal plaster splint is a complete circular cast for distal radius fractures. It should be moulded to a three point system against the volar aspect of the proximal fragment and the dorsal aspect of the distal fragment. To prevent radial shortening an ideal cast should have the thumb incorporated as far as the interphalangeal joint as in the case of scaphoid fracture. Sir John Charnley further emphasises that although the complete cast is superior, the plaster slab may be more convenient (Figure $1 \& 2$ ).

He emphasized the fact that the plaster slab should not merely be a dorsal slab, but it must always be a radial slab, again with the principles of three point fixation. It is essential that the slab reach the midline of the forearm on the volar aspect and should be thick enough to take a permanent impression from the surgeon's thenar eminence while the plaster is setting.

There are two schools of thoughts regarding the relationship between the anatomical and functional outcome. One school which is supported by Sarmiento et al. ${ }^{8}$ and Green. ${ }^{9}$ stresses the need to obtain and maintain an anatomical reduction as they have demonstrated a direct relationship between the residual deformity and mean disability. The other school, which is supported by Cassebaum. ${ }^{2}$ Dias et al. ${ }^{10}$ and McAuliffe et al. ${ }^{11}$ have claimed that there is no such relationship and that no person who had Colles' fracture more than five years old had any serious functional complaints. In a review entitled "Clinical Policies on Distal Radius Fractures" produced by the American Academy of Orthopaedic Surgeons (1996), it was stated that the wrist is not a weight-bearing joint and therefore adequate function after healing is not necessarily compromised by residual metaphyseal deformity. McQueen et al. ${ }^{12}$ seems to be in both the groups as on one side he has proved that a deformed wrist results in a weakness and pain but on the other hand has also shown that re-manipulating wrist fractures in the elderly does not gives lasting results and is not justified in this age group.

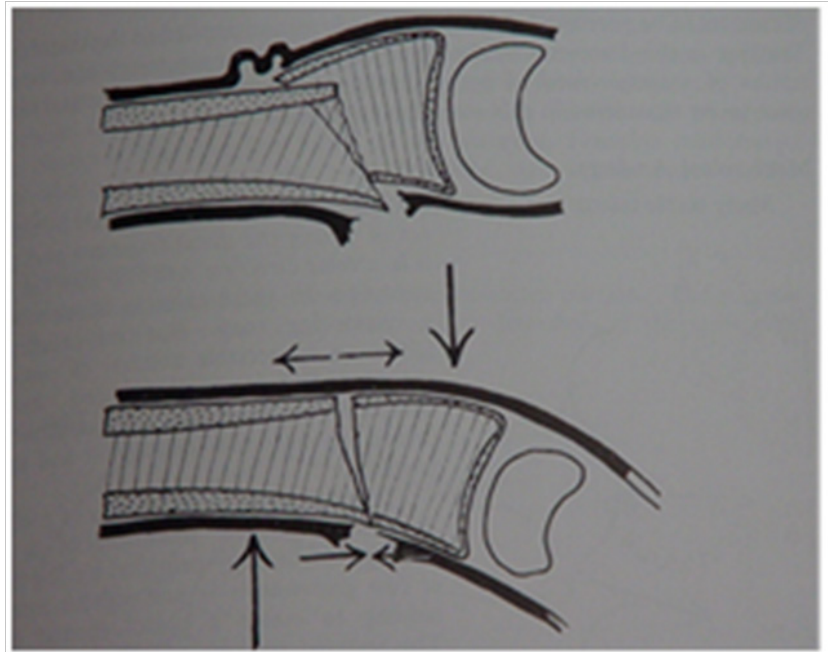

Figure I Diagram illustrating three-point moulding using the intact periosteum. $^{13}$

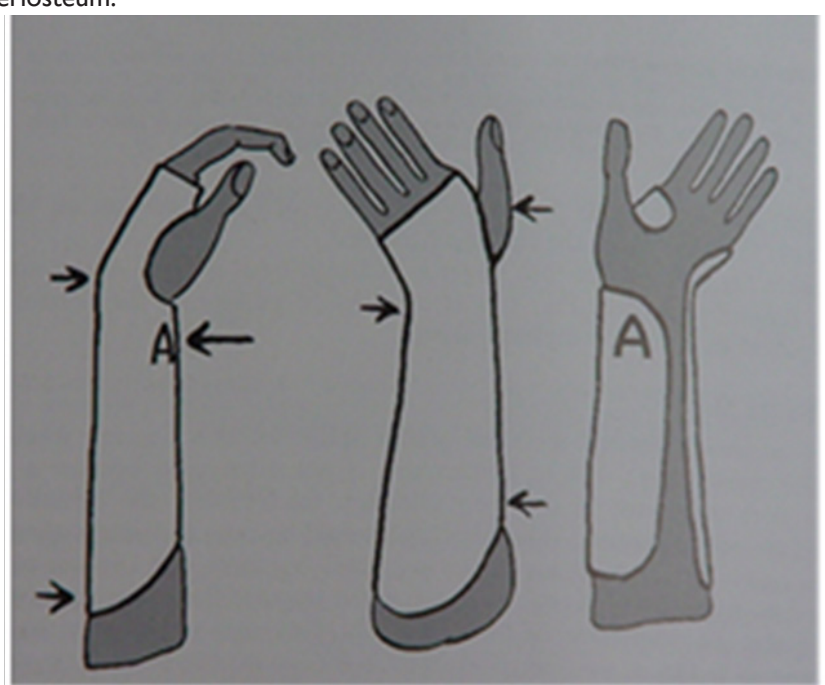

Figure 2 The radial plaster slab. Thick plaster at point $\mathrm{A}$ in order to make the plaster conform to the principles of a three point splint. ${ }^{13}$

We are unable to comment on the relationship of the anatomical and functional outcome as this is one of the weaknesses of our study that we couldn't assess the patients functionally and only had access to the notes and X-rays.

The other weaknesses in our study are that we didn't know the normal angles of the patient's wrist, as didn't have X-rays of the 
normal side. Although there were sixty patients treated for wrist fractures according to our protocol we only could examine the notes and X-rays of the forty patients available to us, therefore we couldn't select the patients randomly. Bias should be minimal however, as we had no control on the selection of these patients and examined the records of only those whom we had access too. Lastly there is a potential problem with X-ray magnification. Although there is a set standard of taking antero-posterior and lateral X-rays of the injured wrist in the X-ray department, because of the pain in the injured wrist, there is no absolute control on the position of the wrist lying on the $\mathrm{X}$-ray film, and it can be highly dependent on the patient's threshold for pain.

\section{Conclusion and recommendations}

Although distal radial fractures in elderly can be reduced easily under Bier's block, it is extremely difficult to maintain their position as more than $50 \%$ of our cases lost reduction. It is likely that this high failure rate is due to osteopenia and combination, as Abbaszadegan. ${ }^{5}$ has shown that the instability of a distal radius fracture increases with age and metaphyseal combination both of which imply osteopenia.

We do not agree with Beamer \& McQueen. ${ }^{6}$ who believe that reduction of fractures of the distal radius is ineffective. In most of our patients a good reduction was achieved after manipulation and in 26 out of the 30 patients who had cast completed within 24 hours, the position was accepted as the final outcome.

Lastly we would stress on the fact that plaster slab or cast, whichever is convenient for the staff, should be placed according to the recommendations of Sir John Charnley. ${ }^{13}$ as we have seen in our study that the ten patients who were left in the dorsal slab, after manipulation, for more than twenty four hours, lost reduction and all of them needed a further operation.

\section{Acknowledgments}

None.

\section{Conflicts of interest}

None.

\section{References}

1. French RJ Fractures and Dislocations of the Wrist. In: Brinker (Ed.), Review of Orthopaedic Trauma. WB Saunders Company, USA, 2001;21:277-302.

2. McQueen M, Caspers J Colles' fracture: Does the anatomical result affect the final function? J Bone Joint Surg. 1988;70(4):649-651.

3. Gupta A The treatment of Colles' fracture: Immobilisation with the wrist dorsiflexed. J Bone Joint Surg 1991;73(2):312-315

4. Zabinski SJ, Weiland AJ Fractures of the distal radius. Orthopaedic Knowledge. 1996;7:67-82.

5. Abbaszadegan H, Jonsson U External fixation or plaster cast for severely displaced Colles' fracture? Prospective 1-year study of 46 patients. Acta Orthopaedic Scandinavica. 1990;61(6):528-530.

6. Beumer A, McQueen M Fractures of the distal radius in low-demand elderly patients; Closed Reduction of no value in 53 of 60 wrists. Acta Orthopaedic Scandinavica. 2003;74(1):98-100.

7. Van der Linden W, Ericson R Colles' fracture. How should its displacement be measured and how should it be immobilized? $J$ Bone Joint Surg. 1981;63(8):1285-1288.

8. Sarmiento A, Pratt GW, Berry NC et al. Colles' fractures: functional bracing in supination. J Bone Joint Surg. 1975;57(3):311-317.

9. Green DP Pins and plaster treatment of commented fractures of the distal end of the radius. J Bone Joint Surg. 1975;57(3):304 -310.

10. Dias JJ, Wray CC, Jones JM, Gregg PJ The value of early mobilisation in the treatment of Colles' fractures. J Bone Joint Surg. 1987;69(3):463-467.

11. McAuliffe TB, Hilliar KM, Coates CJ et al. Early mobilisation of Colles' fractures: a prospective trial. J Bone Joint Surg. 1987;69(2):727-729.

12. McQueen M, MacLaren A, Chalmers J The value of remanipulating Colles' fractures. J Bone Joint Surg. 1986;68(2):232-233.

13. John Charnley The Closed Treatment of Common Fractures. (4th edn), Cambridge University Press, USA, pp. 2011;1-184. 